\title{
ORIGINAL ARTICLE \\ CCL-2 as a possible early marker for remission after traumatic spinal cord injury
}

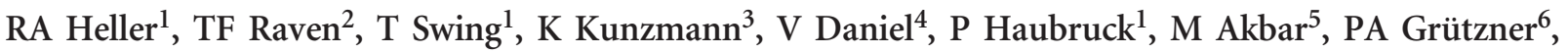 \\ G Schmidmaier $^{1}$, B Biglari ${ }^{6}$ and A Moghaddam ${ }^{2}$
}

\begin{abstract}
Study design: Prospective observational study.
objectives: To describe the correlation between CCL-2, CCL-3, CCL-4 and CXCL-5 serum levels and remission after traumatic spinal cord injury (SCl) in a human protocol compared with animal studies.

Setting: Germany, Rhineland-Palatinate (Rheinland-Pfalz).

Methods: We examined the serum levels of CCL-2, CCL-3, CCL-4 and CXCL-5 over a 12-week period; in particular, at admission and 4,9 and $12 \mathrm{~h}, 1$ and 3 days and 1,2, 4, 8 and 12 weeks after trauma. According to our study design, we matched 10 patients with $\mathrm{TSCl}$ and neurological remission with 10 patients with an initial ASIA A grade and no neurological remission. In all, 10 patients with vertebral fracture without neurological deficits served as control. Our analysis was performed using a Luminex Cytokine Panel. Multivariate logistic regression models were used to examine the predictive value with respect to neurological remission vs no neurological remission.

Results: The results of our study showed differences in the serum expression patterns of CCL-2 in association with the neurological remission (CCL-2 at admission $P=0.013$ ). Serum levels of CCL-2 and CCL-4 were significantly different in patients with and without neurological remission. The favored predictive model resulted in an area under the curve (AUC) of $93.1 \%$ in the receiver operating characteristic (ROC) analysis.

Conclusions: Our results indicate that peripheral serum analysis is a suitable concept for predicting the patient's potential for neurological remission after TSCl. Furthermore, the initial CCL-2 concentration provides an additional predictive value compared with the NLI (neurological level of injury). Therefore, the present study introduces a promising approach for future monitoring concepts and tracking techniques for current therapies. The results indicate that future investigations with an enlarged sample size are needed in order to develop monitoring, prognostic and scoring systems.
\end{abstract}

Spinal Cord (2017) 55, 1002-1009; doi:10.1038/sc.2017.69; published online 20 June 2017

\section{INTRODUCTION}

Spinal cord injury (SCI) remains one of the most challenging impairments in modern medicine. SCIs and resulting paraplegia severely afflict patient's quality of life and cause financial problems for both patients and their families. ${ }^{1-3}$ Besides surgical treatment and rehabilitation, currently no direct treatment for traumatic SCI, such as medication, exists, thus, limiting the success of available treatments and leaving a high risk of an unsatisfactory outcome ${ }^{4-6}$ furthermore, evidence regarding most therapeutic strategies is scarce. ${ }^{6,7}$ Up to date, there is no valid marker able to indicate a possible potential for remission. Therefore, further information on the biochemical processes subsequent to SCI is needed. ${ }^{8}$ In previous studies, ${ }^{8,9}$ we were able to establish the serum cytokine analysis as a valid method for investigating the biochemical processes following SCI by showing characteristic serum level patterns of different cytokines (interferon- $\gamma$, tumor necrosis factor- $\alpha$, interleukin-1 $\beta$, IL- 6 , IL-8, IL-10 and vascular endothelial growth factor) associated with the severity of the neurological impairment and the neurological remission after traumatic SCI.

The pathophysiological process after SCI is complex and consists of a first and second phase that is related to the mechanical trauma and subsequently a variety of pathophysiological processes. During the subacute phase, the inflammatory response includes an initial wave of infiltrating neutrophils. Those are followed by a migration of monocytes and macrophages into the injured segment through various mechanisms. CXCLs (C-X-C motif chemokines) and CCLs (chemokine ( $\mathrm{C}-\mathrm{C}$ motif) ligands) are known for their crucial function in the recruitment of monocytes within the secondary phase of injury after SCI, thereby having a substantial impact on neurological remission. Furthermore, both have well-established roles in the control of the leukocyte recruitment to local inflammation sites. ${ }^{10,11}$ Central nervous system (CNS) Injury is known to trigger hepatic CC and CXC chemokine expression, and thus they appear to amplify the central nervous system response to injury. Furthermore, in 2005

${ }^{1}$ Heidelberg Trauma Research Group, Trauma and Reconstructive Surgery, Center for Orthopedics, Trauma Surgery and Spinal Cord Injury, Heidelberg University Hospital, Heidelberg, Germany; ${ }^{2}$ Aschaffenburg Trauma and Orthopaedic Research Group, Center for Trauma Surgery, Orthopedics and Sports Medicine Aschaffenburg-Alzenau, Aschaffenburg, Germany; ${ }^{3}$ Institute for Medical Biometry and Informatics, Heidelberg University Hospital, Heidelberg, Germany; ${ }^{4}$ Transplantation Immunology, Institute of Immunology, University of Heidelberg, Heidelberg, Germany; ${ }^{5}$ Spine Center, Center for Orthopedics, Trauma Surgery and Spinal Cord Injury, Heidelberg University Hospital, Heidelberg, Germany and ${ }^{6}$ Department of Paraplegiology, Berufsgenossenschaftliche Unfallklinik Ludwigshafen, Ludwigshafen, Germany

Correspondence: Dr B Biglari, Department of Paraplegiology, Berufsgenossenschaftliche Unfallklinik Ludwigshafen, Ludwig-Guttmann-Str. 13, 67071 Ludwigshafen, Germany. E-mail: bahram.biglari@bgu-ludwigshafen.de

Received 2 January 2017; revised 8 May 2017; accepted 8 May 2017; published online 20 June 2017 
Table 1 Demographic and clinical characteristics of patients with $\mathrm{TSCl}$ (Table 1a) and referring to the control group C (Table 1b)

\begin{tabular}{|c|c|c|c|}
\hline (a) Patients & Remission (G1) & $\begin{array}{l}\text { No remission } \\
\text { (GO) }\end{array}$ & $\begin{array}{c}\text { Comparison G1 GO } \\
\text { (P-value) }\end{array}$ \\
\hline$N$ & 10 & 10 & \\
\hline Age (years) & $40 \pm 22$ & $42 \pm 17$ & 0.623 \\
\hline $\begin{array}{l}\text { Time accident/alarm to } \\
\text { admission (min) }\end{array}$ & $71 \pm 32$ & $73 \pm 22$ & 0.752 \\
\hline Duration 1st OP (min) & $154 \pm 59$ & $198 \pm 84$ & 0.205 \\
\hline \multicolumn{4}{|l|}{ Gender } \\
\hline Female & 3 & 1 & 0.264 \\
\hline Male & 7 & 9 & \\
\hline \multicolumn{4}{|l|}{ Etiology } \\
\hline Fall & 5 & 4 & 0.574 \\
\hline Traffic & 5 & 5 & \\
\hline Other & 0 & 1 & \\
\hline \multicolumn{4}{|l|}{ Paralysis } \\
\hline Incomplete paraplegia & 4 & 0 & 0.067 \\
\hline Complete paraplegia & 3 & 4 & \\
\hline Incomplete tetraplegia & 3 & 3 & \\
\hline Complete tetraplegia & 0 & 3 & \\
\hline None & 0 & 0 & \\
\hline \multicolumn{4}{|l|}{ AO classification } \\
\hline A & 7 & 6 & 0.320 \\
\hline B & 3 & 2 & \\
\hline C & 0 & 2 & \\
\hline \multicolumn{4}{|l|}{$N L I$} \\
\hline C & 3 & 6 & 0.343 \\
\hline Th & 4 & 3 & \\
\hline $\mathrm{L}$ & 3 & 1 & \\
\hline None & 0 & 0 & \\
\hline \multicolumn{4}{|l|}{ AIS at admission } \\
\hline A & 3 & 10 & \\
\hline $\mathrm{B}$ & 2 & 0 & \\
\hline C & 5 & 0 & \\
\hline $\mathrm{D}$ & 0 & 0 & \\
\hline$E$ & 0 & 0 & \\
\hline \multicolumn{4}{|l|}{ AIS after 12 weeks } \\
\hline A & 0 & 10 & \\
\hline $\mathrm{B}$ & 1 & 0 & \\
\hline C & 3 & 0 & \\
\hline $\mathrm{D}$ & 6 & 0 & \\
\hline$E$ & 0 & 0 & \\
\hline
\end{tabular}

(b) Patients

Control (C)

$$
\text { N }
$$

Age (years)

10

Gender

Female

Male

Etiology

Fall

Traffic

Other

AO classification

A

B

C
Campbell et al. ${ }^{10}$ showed in a rodent model that artificial elevation of circulating CCL-2 alone was sufficient to accelerate the rate of monocyte recruitment to the injured CNS while dexamethasone significantly reduced hepatic CCL-2 mRNA, leucocytosis and leukocyte recruitment.

In this study, we sought to determine a prognostic model for the evaluation of the remission potential after SCI based on potential biomarkers CXCLs and CCLs in peripheral blood serum. ${ }^{12}$ Due to their relevance in (neuro-) inflammatory processes, in particular after traumatic injuries, we decided to investigate on the serum levels of CCL-2, CCL-3, CCL-4 and CXCL-5. ${ }^{13-16}$ The results of this study intend to provide a novel possible diagnostic method to help predicting the potential remission after SCI and evaluating clinical therapies regarding their success on remission levels. ${ }^{17,18}$ Therefore, this study focused on the following research questions:

(1) Is it possible to predict rehabilitation after SCI based on serum cytokine expression levels?

(2) Is it possible to use analysis of the serum chemokine expression levels as a possible diagnostic tool for monitoring neurological remission in future therapies after TSCI?

The answers to these questions will help us to come closer to monitoring improvement as well as developing an objective score in predicting the remission after SCI.

\section{MATERIALS AND METHODS}

From 2011 to 2016, 141 patients (103 males and 38 females) concerned by TSCI and treated in the Berufsgenossenschaftliche Unfallklinik Ludwigshafen (BG Trauma Centre) were included prospectively in our register. Acquisition of blood samples took place in each case with a time accuracy of $\pm 10 \%$ : four vials of serum (each $7.5 \mathrm{ml}$ ) were obtained at 4, 9 and $12 \mathrm{~h}, 1$ and 3 days and 1, 2, 4, 8 and 12 weeks after SCI. After $20 \mathrm{~min}$ of coagulation, blood samples were centrifuged at $3000 \mathrm{rpm}$, aliquoted and stored at $-80^{\circ} \mathrm{C}$ until analysis.

According to the respective status of the database, different collections of serum samples have been analyzed to address the respective scientific issues. ${ }^{8,9,19-24}$ Assessment of the AIS grades took place at the admission as well as after 12 weeks in accordance with the International Standards for Neurological Classification of SCI (ISNCSCI). ${ }^{25}$ The criterion for neurological remission was defined as positive conversion of AIS grades within 12 weeks after trauma. All ISNCSCI examinations were performed by the head physical therapist at the BG Trauma Center. Initial ISNCSCI examinations were performed within the first $72 \mathrm{~h}$ after admission. Patients recruited for the register were admitted within two hours after activation of the emergency response system ${ }^{8}$ at the BG Trauma Center, a primary trauma center with its own emergency helicopter. Surgical treatment then was realized within $3.52 \pm 1.35 \mathrm{~h}$ after trauma. The GLP (Good Laboratory Practice) provisions set the standard for all examinations of serum samples. Luminex Performance Human High-Sensitivity Cytokine Panels were used for the quantitative determination of CCL-2, CCL-3, CCL-4 and CXCL-5 in the patients' serum samples. Determination was performed according to the manufacturer's instructions (Catalog Number FCST03-04, Kit Lot Number 1420835). The kits were provided by R\&D Systems (Minneapolis, MN, USA). The respective analytes' characteristics are the following given in sensitivity ( $\mathrm{pg} \mathrm{ml}^{-1}$ ) and standard curve range (pg ml$\left.{ }^{-1}\right)$ : CCL-2 (9.9; 98-7940), CCL-3 (16.2; 181-14 700), CCL-4 (5.8; 261-21 150) and CXCL-5 (8.2; 50-12 140). All quantitative analyses was carried out in the Heidelberg University Hospital by a lab technician blinded to both patients and clinical information.

The present study has been approved by the ethics committee of the University of Heidelberg (S-514/2011) and the Landesärztekammer RheinlandPfalz (837.188.12/8289-F), Germany. The study is registered in the German Clinical Trials Register DRKS (Study-ID: DRKS00009917/Date of Registration: 23.03.2016/Universal Trial Number (UTN): U1111-1179-1620).
Age is expressed as mean years \pm s.d. Neurological remission was defined as improvement in
AIS. Comparing GO and G1, P-values were analyzed with either the $\chi^{2}$ test or Mann-Whitne $U$-test, and they show differences between GO and G1. 
Consent forms of all study participants were signed and dated willingly. All patients could voluntarily choose to leave the study at any time and for any reason. Patients matching the exclusion criteria (nontraumatic spinal cord injury, traumatic brain injury (TBI), severe abdominal trauma, traumatic amputation of extremities, coma and additional major trauma apart from the SCI) were excluded without exception. None of the patients received methylprednisolone sodium succinate (MPSS) during study participation.

\section{Matching}

In order to reduce the influence of clinical covariates on the respective cytokine level, groups of matched pairs were set up to investigate differences within their cytokine pattern. Therefore, we matched 10 patients with SCI and neurological remission (Group 1=G1) with 10 patients with an initial American Spinal Injury Association (ASIA) A grade and no neurological remission (Group $0=\mathrm{G} 0$ ). Furthermore, 10 patients with an isolated vertebral fracture without neurological deficits served as a control group (Group $\mathrm{C}=\mathrm{C}$ ).

Matching criteria were the following: age, sex, etiology and AO ("Arbeitsgemeinschaft für Osteosynthesefragen"; German for "Association for the Study of Internal Fixation") fracture classification. Given the case that more than one match was allocable, then the patient with the most comparable clinical profile was preferred and vice versa. The resulted patients' demographics are given in Tables 1 and 2 shows the AO classification ${ }^{26}$ of each patient in group C. The matching procedure was performed retrospectively based on the clinical data. Patient enrollment, sample collection and analysis of CCL-2, CCL-3, CCL-4 and CXCL-5 were performed in a prospective manner.

\section{Statistical analysis}

Explorative correlation analyses were conducted between all variables. Nonparametric test methods were assessed to investigate location shifts between (Mann-Whitney $U$-test) as well as within groups at different time points (Wilcoxon Signed Rank Test). More than two independent samples were analyzed using the Kruskal-Wallis test. Categorical variables were evaluated using the $\chi^{2}$ test.

The respective predictive power of variables regarding the criterion neurological remission, a positive AIS conversion within 12 weeks after injury, was assessed via logistic regression modeling. Clinical relevance of covariates was additionally considered. Model selection was performed via AIC (Akaike Information Criterion $)^{27}$ comparison. Predictive performance was assessed by estimation of the AUC (area under the curve) of the ROC (receiver operating characteristic) Curve and the respective confidence interval. All $P$-values quoted are to be interpreted in a descriptive way as they were not adjusted for multiple testing as this is an exploratory post hoc analysis. All statistical calculations were performed with $\mathrm{R}$ version $3.2 .3^{28}$ and 'pROC'29 for receiver operator characteristics (ROC) analysis. Figures were created by using the package 'ggplot2'.30

\section{Related work}

The register has been addressed for several scientific issues. ${ }^{8,9,19-24}$ The respective studies are drawn from different data sets. Analyses were performed on the respective status of the database and therefore include differing numbers

Table 2 AO classification of the control group (group C)

\begin{tabular}{lc}
\hline$I D$ & $A O$ \\
\hline 2 & $52.12: \mathrm{A} 3.3$ \\
24 & $52.11: \mathrm{A} 3.3$ \\
33 & $53.2: \mathrm{A} 3.2$ \\
37 & $53.2: \mathrm{A} 3.3$ \\
63 & $52.7-10: \mathrm{A} 1.1$ \\
65 & $52.12: \mathrm{A} .2$ \\
67 & $52 / 3.12-1: \mathrm{A} 3.1$ \\
89 & $52.12: \mathrm{A} 3.1$ \\
92 & $52.3-4: \mathrm{B} 2.1$ \\
93 & $53.3: \mathrm{A} 1.2$ \\
\hline
\end{tabular}

of patients according to the respective inclusion criteria as well as different matched patients due to the growing variety within the database. Some patients were enrolled in more than one study. All samples were collected in a prospective manner. Our recent investigation of matrix metalloproteinases ${ }^{22}$ shares a similar explorative approach of matching procedure and data analysis. The study at hands shares some clinical data of patients who were enrolled in both works.

\section{RESULTS}

The present study was designed as a prospective and explorative clinical observer study. Patients included into this study were matched out of 141 cases in our database. There was no randomization. ${ }^{31}$ Criteria for the match-pair analysis contained the patient's sex, age, etiology and AO classification (Table 1). ${ }^{32}$ The patients' demographics were documented; analysis and comparison of groups were performed as previously published. ${ }^{21-24}$

\section{Patients demographics}

According to our established matched-pair analysis, 30 patients were included into this study 22 (9 females and 21 males) out of our present database counting 141 cases that were admitted from 2011 until 2016. The mean age was $42 \pm 17$ years. The study group (Group S) consisted of 20 patients who were neurologically affected by TSCI. The control group (Group C) was composed of 10 patients who had a traumatic injury without neurological impairment. In group S, there were 10 with (Group 1 remission) and 10 without AIS improvement (Group 0 no remission). Those without AIS improvement were chosen to have an initial AIS grade A. In C, there were no lesions of the spinal cord. All clinical characteristics are given in Table 1. Additionally, Table 3 shows the particular development of AIS grades in G1.

Statistically, there was no difference between study groups regarding age, timespan from accident/alarm to admission, duration of the 1st operation, gender, etiology and AO classification (referred to as G1 and G0 in the following text). All 20 patients with traumatic SCI (G0 and G1) received surgery (8 ventral $40.0 \% ; 12$ dorsal $60.0 \%$ ); 15 were treated with spondylodesis $(75.0 \%)$. All 30 patients included in the collective suffered vertebral fractures. The AIS grades at admission and discharge as well as the NLI and the type of paralysis were significantly different in G1 and G0 $(P<0.05)$.

\section{Analysis of the entire patient collective}

In this study, we investigated whether and how CXCL and CCL serum values reflect the biochemical processes after SCI by comparing patients with (S) neurological impairment to patients without (C) neurological impairment as well as patients without remission (G0) compared with patients with remission (G1). Comparison between groups of the serum expression patterns of CXCL $(\mathrm{C}-\mathrm{X}-\mathrm{C}$ motif chemokine) and CCL (chemokine ( $\mathrm{C}-\mathrm{C}$ motif) ligand) is shown in

Table 3 AIS Conversion of patients with neurological remission (G1) between admission and 12 weeks after injury

\begin{tabular}{|c|c|c|c|c|}
\hline \multirow[t]{2}{*}{ AIS grade } & \multicolumn{2}{|c|}{ AIS at admission } & \multicolumn{2}{|c|}{ AIS after 12 weeks } \\
\hline & $\mathrm{N}$ & $\%$ & $\mathrm{~N}$ & $\%$ \\
\hline A & 3 & 33.3 & 0 & 0.00 \\
\hline$B$ & 2 & 20.0 & 1 & 10.0 \\
\hline C & 5 & 50.0 & 3 & 33.3 \\
\hline$D$ & 0 & 0.00 & 6 & 66.6 \\
\hline
\end{tabular}

Percentage was calculated by dividing the number of each cell by the sum of column $\left(N_{\mathrm{G} 1}=10\right)$. 
Figures $1 \mathrm{a}-\mathrm{d}$. There were no significant correlations in chemokine serum levels in regard to gender, age, paralysis, $\mathrm{AO}$ classification and etiology or NLI.

CCL-2 within the first $12 \mathrm{~h}$, mean CCL-2 values were higher in G0 than in G1 and C. Serum levels in G0 fell from $702.12 \pm 151.54 \mathrm{pg} \mathrm{ml}^{-1}$ to $394.21 \pm 51.61 \mathrm{pg} \mathrm{ml}^{-1} 4 \mathrm{~h}$ after trauma. In G1, mean CCL-2 values showed a minimum $254.27 \mathrm{pg} \mathrm{ml}^{-1}$ at $12 \mathrm{~h}$ and peak serum levels $425.99 \mathrm{pg} \mathrm{ml}^{-1} 24 \mathrm{~h}$ after trauma. Serum levels in G1 were lower than in G0 throughout the time course, except during peak levels in G1 $24 \mathrm{~h}$ after trauma. In group C, mean CCL-2 levels were lower than those of G0 and G1 during the complete course of time; serum levels showed a decreasing trend from initially $322.41 \pm 146.17 \mathrm{pg} \mathrm{ml}^{-1}$ to $140.14 \mathrm{pg} \mathrm{ml}^{-1}$ two weeks after trauma. Differences between G0 and G1 at admission $(P=0.013)$ and 2 months $(P=0.050)$ after trauma were significant when analyzed with the Mann-Whitney $U$-test. Furthermore, results of our study showed a significant initial elevation of CCL-2 in G1 compared with that in G0 $(P=0.013)$. Similar to CCL-3 and and CCL-4, CCL-2 expression levels showed an increasing trend from $12 \mathrm{~h}$ to 3 days after TSCI in G0 and G1.

CCL-3. Mean CCL-3 serum levels of all patients started within the range from 81.72 to $128.26 \mathrm{pg} \mathrm{ml}^{-1}$. Those of $\mathrm{G} 0$ and $\mathrm{C}$ stayed at a consistent level. At a duration of $9 \mathrm{~h}$ after trauma in G1, CCL-3 values rose from $86.58 \pm 9.84 \mathrm{pg} \mathrm{ml}^{-1}$ to the maximum of $174.55 \pm 56.42 \mathrm{pg} \mathrm{ml}^{-1}$ at $24 \mathrm{~h}$ and fell again to $78.95 \pm 13.69 \mathrm{pg} \mathrm{ml}^{-1}$ 1 week after trauma. The huge $\mathrm{CI}$ at the maximum level in G1 was mostly driven by one single and low value of $32.34 \mathrm{pg} \mathrm{ml}^{-1}$. Mean levels of all G0 and G1 concluded within the range from $81.57 \mathrm{pg} \mathrm{ml}^{-1}$ to $128.69 \mathrm{pg} \mathrm{ml}^{-1}$ after 3 months, and mean levels in $\mathrm{C}$ finally remained on a level of $71.95 \pm 21.63 \mathrm{pg} \mathrm{ml}^{-1} 1$ month after

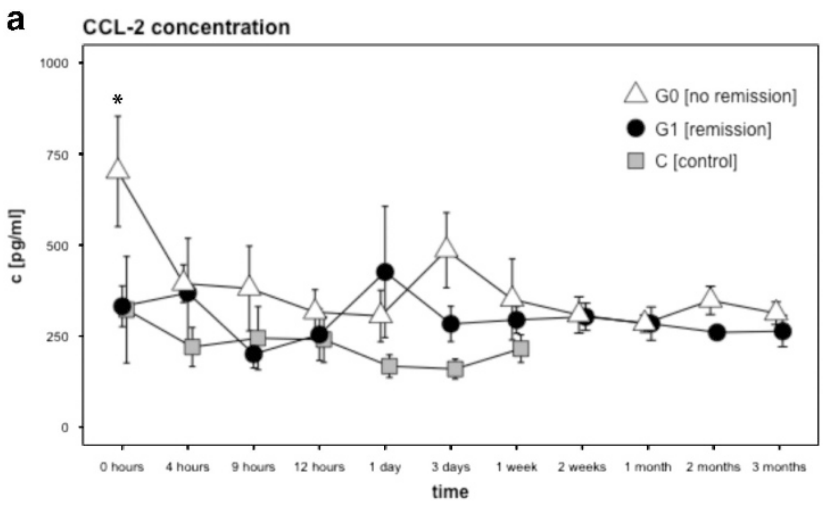

b

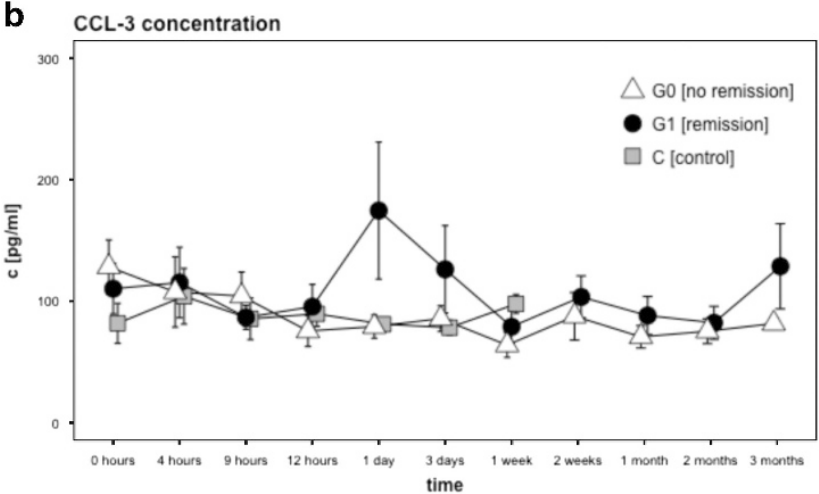

trauma. Analysis with the Mann-Whitney $U$-test showed nonsignificant differences between groups G0 and G1 at any time points.

CCL-4. The analysis of CCL-4 serum levels revealed a similar expression pattern to CCL-3. Mean CCL- 4 values of patients started within the range from 71.26 to $176.19 \mathrm{pg} \mathrm{ml}^{-1}$. Those of $\mathrm{G} 0$ and $\mathrm{C}$ remained on a consistent level. At a duration of $9 \mathrm{~h}$ after trauma in G1, CCL-4 values rose from $80.52 \pm 12.67 \mathrm{pg} \mathrm{ml}^{-1}$ to the maximum of $218.71 \pm 106.06 \mathrm{pg} \mathrm{ml}^{-1}$ at $24 \mathrm{~h}$ and fell again to $97.12 \pm 15.89 \mathrm{pg} \mathrm{ml}^{-1} 1$ week after trauma. Mean serum levels of G0 and G1 concluded within the range from 87.63 to $105.17 \mathrm{pg} \mathrm{ml}^{-1}$ after 3 months, and mean levels in $\mathrm{C}$ remained on a consistent level of $33.16 \pm 12.01 \mathrm{pg} \mathrm{ml}^{-1} 1$ month after trauma. In group $\mathrm{C}$, mean CCL-4 serum levels were lower than those of G0 and G1 at every time. Analysis with the Mann-Whitney $U$-test showed significant differences $9 \mathrm{~h}(P=0.046)$ and 2 months $(P=0.029)$ after trauma between G0 and G1. Here, mean G0 and G1 levels were higher throughout the entire 3 months after TSCI than in C. Similar to the expression pattern of CCL-3, mean levels in G1 rose to the maximum at $24 \mathrm{~h}$, indicating higher levels to provide a beneficial effect on TSCI remodeling processes.

CXCL-5. The expression pattern of mean CXCL-5 values of all subgroups G0, G1 and C showed a similar pattern. Starting within the range from 732.70 to $1047.01 \mathrm{pg} \mathrm{ml}^{-1}$, they all reached their maximum 2 weeks after trauma in the range from 1500.01 to $1899.82 \mathrm{pg} \mathrm{ml}^{-1}$. Final mean values in G0 and G1 after 3 months (G1: $1632.41 \pm 272.22 \mathrm{pg} \mathrm{ml}^{-1} ; \mathrm{G} 0: 912.56 \pm 263.05 \mathrm{pg} \mathrm{ml}^{-1}$ ) and in $\mathrm{C}$ after 2 weeks (C: $1500.92 \pm 435.82 \mathrm{pg} \mathrm{ml}^{-1}$ ) were higher than those in the beginning (G1: $1047.01 \pm 173.20 \mathrm{pg} \mathrm{ml}^{-1}$; G0: $732.70 \pm 112.53 \mathrm{pg} \mathrm{ml}^{-1}$; C: $\left.926.51 \pm 222.97 \mathrm{pg} \mathrm{ml}^{-1}\right)$. Analysis with
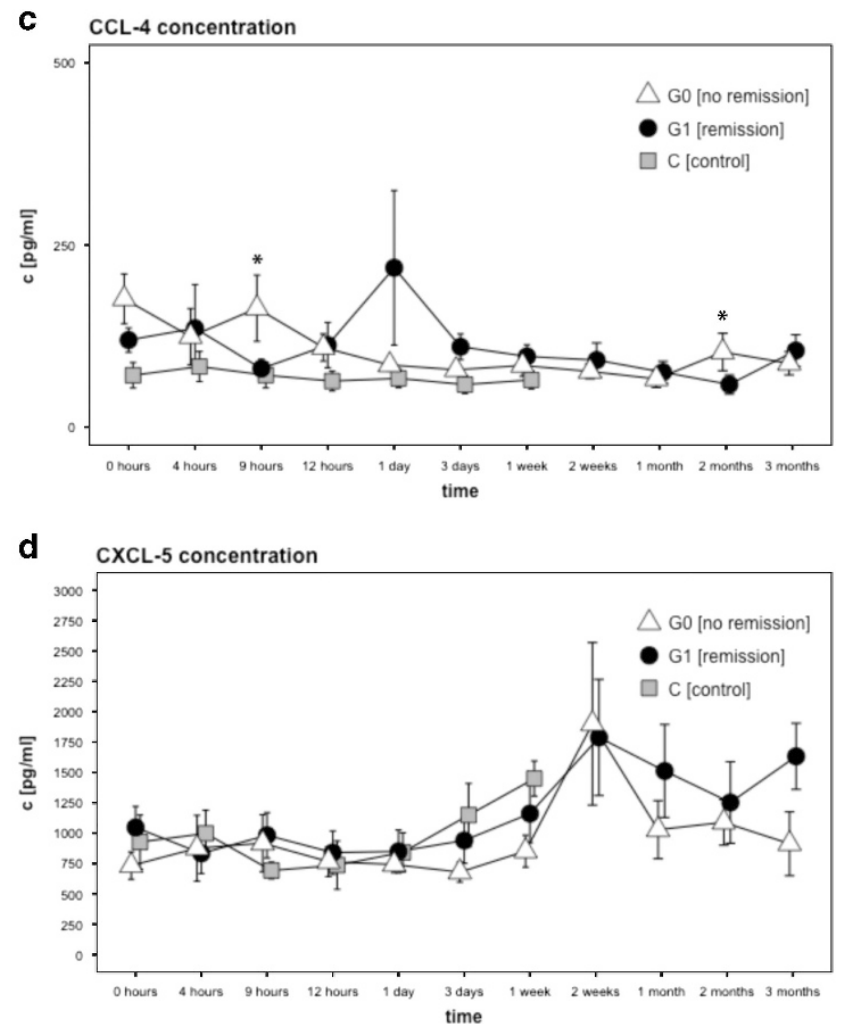

Figure 1 Serum expression patterns of G0, G1 and C expressed as means \pm s.e. of the mean and Serum level comparison of G0 and G1. Differences were assessed with the Mann-Whitney $U$-test between both groups at each particular time point, ${ }^{*} P<0.05$. 
the Mann-Whitney $U$-test showed nonsignificant differences between groups G0 and G1 at any time points.

\section{Binary logistic model}

We utilized binary logistic regression models regarding differences of cytokine blood level patterns in the univariate analysis to compile a predictive model for the potential of remission. Patients with incomplete data points were excluded from this analysis. Thus, 17 out of 20 patients of group $S$ were included in the modeling process investigating remission vs no remission. Variables included were standardized. All initial available clinical variables (sex, age, time to admission, etiology, NLI and AO) as well as any serum parameters were included in the process. All models considered the NLI as baseline due to its prompt availability, obtainment with minimal effort in clinical practice and known association with the outcome. Only this way, it was possible to assess the additional predictive power of the remaining potential covariates.

Based on AIC, the best performing model was the one using only NLI and CCL-2 serum concentration at admission as predictors. Using this model to predict G0_G1 resulted in an AUC of 0.93 (CI: 0.81-1.00). The 95\% confidence intervals and $P$-values for the adjusted odds ratios are given in Table 4 . The ROC is depicted in Figure 2. The significant impact of h0_CCL-2 on the odds of an improvement indicates that this serum parameter does indeed have additional predictive power over using the NLI alone.

\section{DISCUSSION}

In this study, we sought to determine a prognostic model for the evaluation of the remission potential after SCI based on potential biomarkers CXCLs and CCLs in peripheral blood serum. The findings of our study provide important information for the initial assessment of TSCI patients. Our data show that differences of CCL-2 between G1 and G0 at admission $(702.12 \pm 151.55$ vs $331.84 \pm 55.84 ; P=0.013)$ and CCL -4 at $9 \mathrm{~h}(80.52 \pm 71.0$ vs $180.06 \pm 47.79 ; P=0.046)$ indicate those patients who are at high risk for a neurological impairment within the first $4 \mathrm{~h}$ after trauma. Furthermore, the results of our study introduce a predictive value regarding the differentiation between patients without a chance of neurological remission and those with increased probability for remission.

\section{Strength and limitations}

Based on the prospective explorative study design with matched pairs, we investigated the expression patterns of CCL-2, CCL-3, CCL-4 and CXCL-5 in human peripheral serum throughout the acute, subacute and intermediate phase of traumatic SCI according to an established protocol. Our large database $(n=141)$ enabled us to perform an accurate match-pair analysis. ${ }^{8,19,20}$ Although results from animal models are limited by their transferability, ${ }^{17}$ the unlimited sample size remains advantageous. The present study included a small collective of 30 patients of matched pairs, and therefore results may

Table 4 The odds ratios of variables included the preferred logistic regression model

\begin{tabular}{lcccc}
\hline Variables & Adj. odds ratio & P-values & \multicolumn{2}{c}{ Confidence interval } \\
\cline { 3 - 5 } & & & $2.50 \%$ & $97.50 \%$ \\
\hline NLI & 2.737798352 & 0.228 & $6.631011 \mathrm{e}-01$ & 24.8544236 \\
h0_CCL-2 & 0.001047554 & 0.043 & $1.068837 \mathrm{e}-07$ & 0.1504267 \\
\hline
\end{tabular}

Abbreviations: NLI, neurological level of injury; hO_CCL-2, initial CCL-2 serum concentration. not represent local processes clearly. We decided to include patients with different levels of spinal cord injury and different classifications in the ASIA score in the remission group. In the no-remission group, only ASIA A patients were included, leaving the bias of possible misleading information. Further studies with a larger sample size should address an inter-group (G1 vs G0) comparison with each AISA grade, as the prognosis often differs considerably. Because of the large standard errors of the mean and the small number of patients, the significant differences as described must be considered carefully. In order to investigate the role of CCLs and CXCLs in the pathophysiology after TSCI furthermore, they should be correlated to established inflammation markers such as Interferon- $\gamma$, Tumor necrosis factor- $\alpha$, Interleukin-1 $\beta$, IL-6, IL-8, IL-10 and vascular endothelial growth factor. Strengths of this study are that the AUC indicates remarkable prognostic opportunities independent of the specific cutoff value that was chosen, even though they were based on a small sample size, which limited the informative value of the multivariate statistical analysis. Furthermore, we were able to present distinctive expression patterns for CCL-2 and CCL-4, indicating their involvement in the second phase of injury with regard to G0 and G1. Both also provide Supplementary information toward the differentiation between $\mathrm{S}$ and $\mathrm{C}$.

CCL-2. CCL-2 (also known as monocyte chemoattractant protein-1 MCP-1) is known to be elevated in the CNS and cerebrospinal fluid of individuals with neuroinflammatory conditions characterized by blood-brain barrier (BBB) disruption and leukocyte infiltration of the CNS. ${ }^{33,34}$ Therefore, we presume CCL-2 also to be involved in ongoing neuroinflammatory processes after traumatic SCI. In 2012, Roberts et al. ${ }^{35}$ presented that CCL-2 induces shuttling of b-catenin between PECAM-1 (platelet endothelial cell adhesion molecule-1) and the adherens junction (AJ) in human brain microvascular endothelial cells such that the AJ and monolayer integrity are transiently disrupted. Furthermore, HIF-1 protein complex activated by hypoxia is known to bind to the HIF-1-binding DNA sequence and activates MCP-1 transcription. These findings of Mojsilovic-Petrovic et al. ${ }^{36}$ suggest that HIF-1 is involved in transcriptional regulation of hypoxiaupregulated expression of chemokines MCP-1 and MCP-5 in astrocytes. This finding corresponds well with the initial phase of acute traumatic SCI due to ischemia in tissues. Leukocyte migration to the injured tissue regarding tissue remodeling is crucial, especially in association with the secondary injury response after SCI; therefore, we believe CCL-2 to substantially contribute during this process.

Although neuroinflammation was mainly studied in EAE (experimental autoimmune encephalomyelitis), and M1 monocytes recruited by CCL-2 outnumbered M2, CCL-2 has been viewed as the sole 'ticket' to the CNS for monocytes. ${ }^{37}$ In 2013, Shechter et al. presented an anatomical separation of recruited M1 and M2 monocytes between the lesion site and the CP (choroid plexus). Furthermore, entry through distinct gates of monocyte-macrophages corresponds to the known M1 and M2 waves at the lesion site. ${ }^{38}$

Mean G0 and G1 CCL-2 levels were higher than those in C (Figure 1a), despite a slight overlap after $9 \mathrm{~h}$, consistent with a contribution to the neuroinflammatory process after neurotrauma. With a high probability of $93.1 \%$, the favored model based on our findings provides higher results in case of a higher probability of neurological remission compared with a low probability (Figure 2; Table 4). The variables given in Table 4 underline the predictive relevance of the initial CCL-2 serum concentration in relation to the NLI. Figure 3 provides detailed insight into the distribution of CCL-2 
concentration at admission regarding the criterion neurological remission.

Findings by Bank et al. ${ }^{39}$ in 2015 underline the importance of investigation in the field of cytokine profiles in patients with TSCI. Especially the examination of the respective pattern of MCP-1 (monocyte chemotactic protein-1, also referred to as CCL-2) and MIP1- $\beta$ (macrophage inflammatory protein- $1 \beta$, also known as CCL-4) was of great interest for us. Both showed significantly differing patterns compared with a healthy control group monitoring the inflammatory process after TSCI. As outlined in their limitations section, they could not provide any information either about the presence or absence of functional recovery or the comparison to an analogous injured control group. Therefore, we strongly suggest the realization of future studies including enlarged sample sizes that provide the ability to address open questions by setting up multivariate regression models. Findings then can easily be compared by future meta-analysis and regression. Moreover, results thus can be adjusted to relevant covariates such as age, sex, ASIA grade monitoring and NLI such as those used by Kwon et al. ${ }^{18}$ They assessed additional predictive value by including IL-6, IL- 8 and CCL-2, here referred as MCP-1, in a logistic multivariate regression model. Our data support their impressing findings regarding the relevance of CCL-2 within the neuroinflammatory process as well as the thereby provided predictive value regarding the patients' outcome. Both studies have to deal with a limited sample size. Resulting bias in this exploratory status can only be diminished by an increased sample size.

In order to gain more specific data and accurate models, further studies with a larger collective are needed. Due to the limited sample size, the present model might be affected by an overfit. We therefore strongly recommend performing cross-validation on models in future studies with a larger collective in order to avoid this bias.

CCL-3. In 2010, Kiguchi et al. ${ }^{40}$ reported that CCL-3 is localized in recruited macrophages using immunohistochemistry. CCL-3 as well as CCL-4 expression pattern show a maximum $24 \mathrm{~h}$ after fracture in G1;

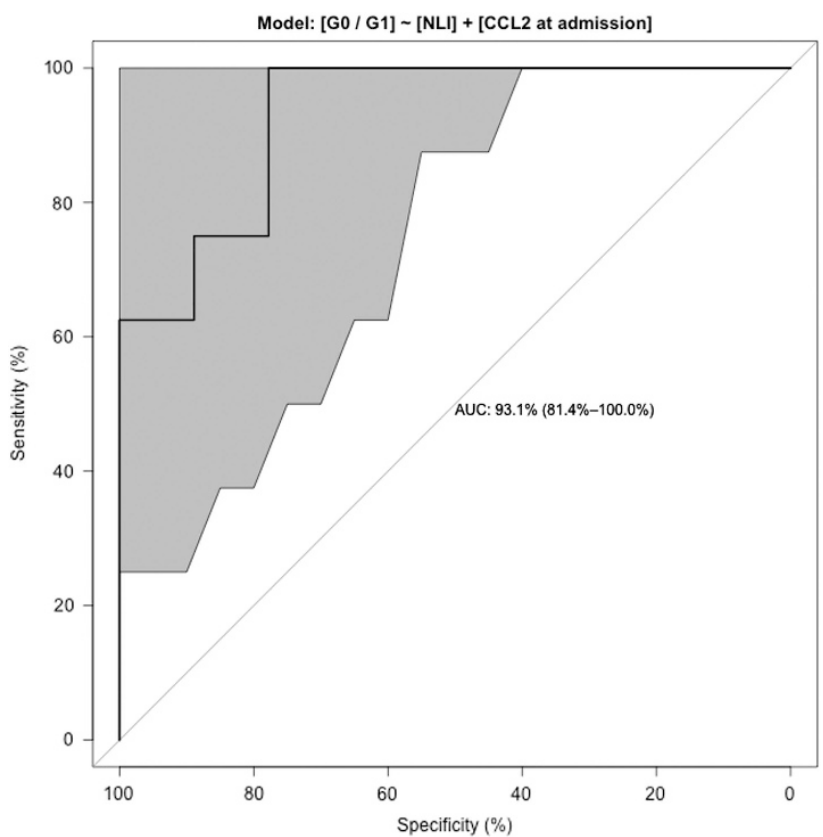

Figure 2 ROCs of the binary regression model. $\mathrm{Cl}$ marked as gray area in both directions. our data are consistent with the neuroinflammatory process being assumed to reach the maximum at $24 \mathrm{~h}$ after trauma.

The huge CI in G1 at $24 \mathrm{~h}$ might be related to the variation within the remodeling process in each patient and reflects the variability of concrete processes. We assume a beneficial effect from elevated CCL-3 levels within that time. For more specific information, further studies are required to examine both the specific origin of CCL-3 as well as the moderating/mediating factors involved.

CCL-4. As key mediators of adhesion to the endothelial surface, chemokines have a major role in the recruitment of T-cell subsets (previously localized to the CNS in vivo) across the BBB during CNS inflammation. Findings of Quandt et al. ${ }^{41}$ in 2010 emphasized the role and importance of CNS-derived CC-chemokines in regulating the traffic of recently activated T-cell subsets across cytokine-activated cerebral endothelium in inflammatory diseases. They reported CCL-4

a

CCL-2 serum concentration at admission

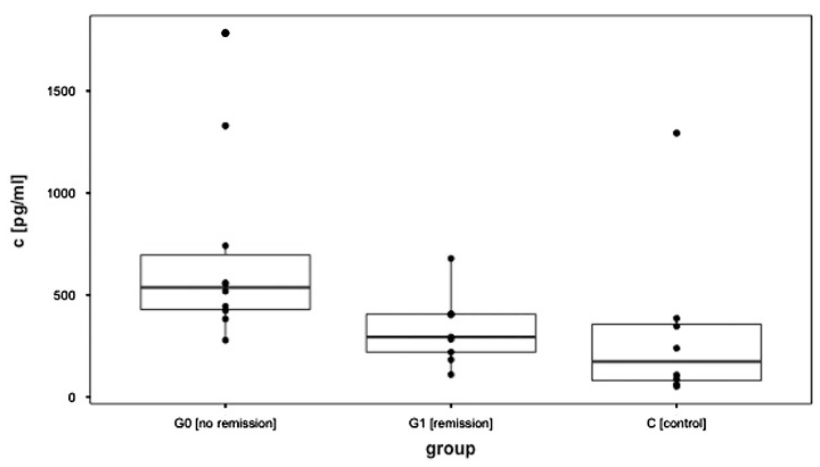

b
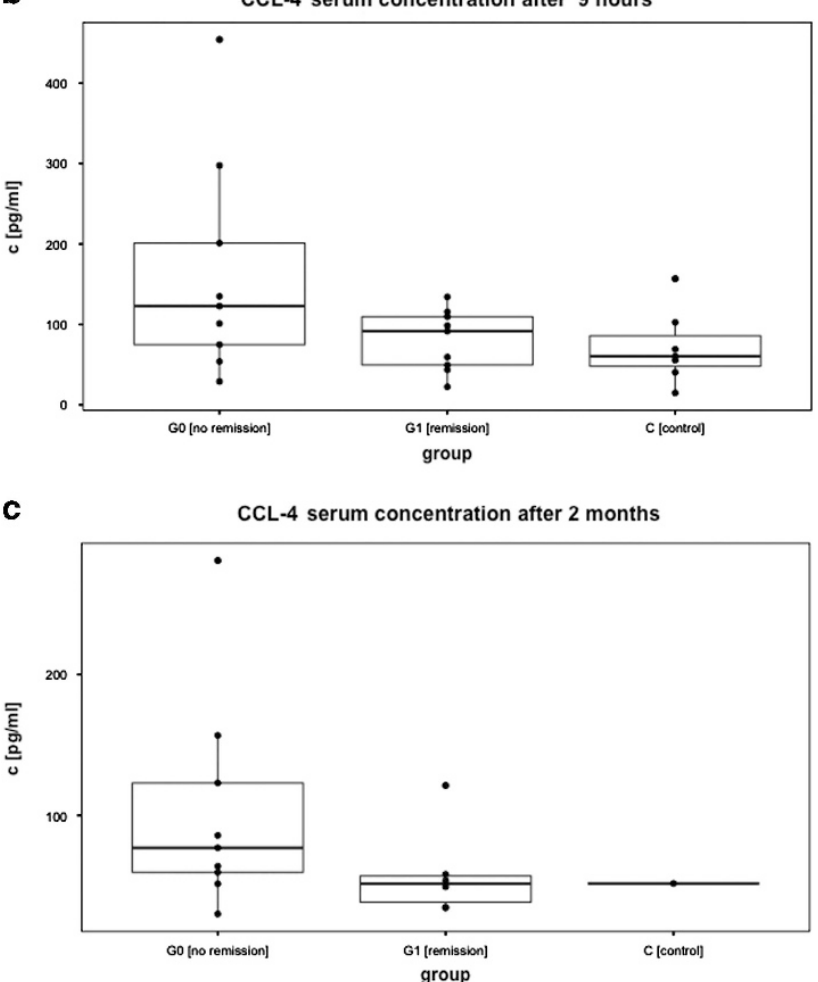

Figure 3 Serum expression levels of CCL-2 at admission and of CCL-4 $9 \mathrm{~h}$ and 2 months after injury. 
and CCL-5 to enhance adhesion of specific CD4+ T-Cell subsets to human brain endothelial cells. Further studies are needed to clarify reasons for the considerable $\mathrm{CI}$ in $\mathrm{Gl}$ at $24 \mathrm{~h}$. Possible explanations might be obtained through classification and phenotypisation of immune cells in the lesion site and complemented by a functional examination. Correlations of CSF, Serum and parenchymal cytokine expression would provide additional information in terms of understanding biochemical processes as well as establishing predicting and monitoring systems.

CXCL-5. Although CXCL-5 is reported to have neuroprotective effects after TSCI, ${ }^{13}$ the results of our study show no further information regarding either identification of an ongoing neurotrauma nor differentiation between G1 and G0. CXCL-5 rather presents a systemic involvement in general recovery processes after trauma. Further studies should therefore focus on CXCL on a local level.

\section{CONCLUSIONS}

Data regarding systemic levels of CCL-2 and CCL-4 derived from peripheral serum analysis provide crucial information about biochemical processes ongoing after TSCI. The predictive model for an improvement in AIS based on NLI and the initial CCL-2 concentration results in an AUC of $93.1 \%$. This means that the chances of assigning a higher model-based score to patients who actually show an improvement as compared with those who do not is 93.1\%. Although the present results should be considered critically due to the small patient collective, they might form the basis for future research toward monitoring and prognostic scoring techniques in TSCI by means of studies with an enlarged sample size.

\section{DATA ARCHIVING}

There were no data to deposit.

\section{CONFLICT OF INTEREST}

The authors declare no conflict of interest.

\section{ACKNOWLEDGEMENTS}

The present study has been funded by the AO foundation with an amount of 6700 SFR. We are grateful for their support. We additionally thank Martina Kutsche-Bauer for performing the Luminex analysis. Statistical consulting was provided by the Insitut for Biometrics and Bioinformatics, University of Heidelberg.

\section{AUTHOR CONTRIBUTIONS}

$\mathrm{AM}$ and $\mathrm{BB}$ conceived and designed the study; $\mathrm{AM}, \mathrm{RAH}$ and $\mathrm{BB}$ performed the patient recruitment and collection of blood samples; VDl contributed materials and analysis tools and was responsible for the measurement of the data; RAH and KK analyzed the data; AM and RAH wrote the paper. MA, TFR, TS, PAG, GS and PH revised the paper.

1 Rowland JW, Hawryluk GW, Kwon B, Fehlings MG. Current status of acute spinal cord injury pathophysiology and emerging therapies: promise on the horizon. Neurosurg Focus 2008; 25: E2.

2 Boakye M, Leigh BC, Skelly AC. Quality of life in persons with spinal cord injury: comparisons with other populations. J Neurosurg Spine 2012; 17: 29-37.

3 Biglari B, Buchler A, Reitzel T, Swing T, Gerner HJ, Ferbert T et al. A retrospective study on flap complications after pressure ulcer surgery in spinal cord-injured patients. Spinal Cord 2014; 52: 80-83.

4 Ito Y, Sugimoto Y, Tomioka M, Kai N, Tanaka M. Does high dose methylprednisolone sodium succinate really improve neurological status in patient with acute cervical cord injury?: a prospective study about neurological recovery and early complications. Spine 2009; 34: 2121-2124.
5 Kwon BK, Okon EB, Plunet W, Baptiste D, Fouad K, Hillyer J et al. A systematic review of directly applied biologic therapies for acute spinal cord injury. J Neurotrauma 2011; 28: 1589-1610.

6 Evaniew N, Belley-Cote EP, Fallah N, Noonan VK, Rivers CS, Dvorak MF. Methylprednisolone for the treatment of patients with acute spinal cord injuries: systematic review and meta-analysis. J Neurotrauma 2015; 33: 468-481.

7 Walters BC, Hadley MN, Hurlbert RJ, Aarabi B, Dhall SS, Gelb DE et al. Guidelines for the management of acute cervical spine and spinal cord injuries: 2013 update. Neurosurgery 2013; 60: 82-91.

8 Moghaddam A, Child C, Bruckner T, Gerner HJ, Daniel V, Biglari B. Posttraumatic inflammation as a key to neuroregeneration after traumatic spinal cord injury. Int $\mathrm{J} \mathrm{Mol}$ Sci 2015; 16: 7900-7916.

9 Biglari B, Swing T, Child C, Buchler A, Westhauser F, Bruckner T et al. A pilot study on temporal changes in IL-1 beta and TNF-alpha serum levels after spinal cord injury: the serum level of TNF-alpha in acute $\mathrm{SCl}$ patients as a possible marker for neurological remission. Spinal Cord 2015; 53: 510-514.

10 Campbell SJ, Perry VH, Pitossi FJ, Butchart AG, Chertoff M, Waters S et al. Central nervous system injury triggers hepatic $C C$ and $C X C$ chemokine expression that is associated with leukocyte mobilization and recruitment to both the central nervous system and the liver. Am J Pathol 2005; 166: 1487-1497.

11 Stanimirovic D, Satoh K. Inflammatory mediators of cerebral endothelium: a role in ischemic brain inflammation. Brain Pathol 2000; 10: 113-126.

12 Yokobori S, Zhang Z, Moghieb A, Mondello S, Gajavelli S, Dietrich WD et al. Acute diagnostic biomarkers for spinal cord injury: review of the literature and preliminary research report. World Neurosurg 2015; 83: 867-878.

13 Tyagi P, Kadekawa K, Kashyap M, Pore S, Yoshimura N. Spontaneous recovery of reflex voiding following spinal cord injury mediated by anti-inflammatory and neuroprotective factors. Urology 2016; 88: 57-65.

14 Lee SI, Jeong SR, Kang YM, Han DH, Jin BK, Namgung U et al. Endogenous expression of interleukin-4 regulates macrophage activation and confines cavity formation after traumatic spinal cord injury. J Neurosci Res 2010; 88: 2409-2419.

15 Brambilla R, Bracchi-Ricard V, Hu WH, Frydel B, Bramwell A, Karmally S et al. Inhibition of astroglial nuclear factor kappaB reduces inflammation and improves functional recovery after spinal cord injury. J Exp Med 2005; 202: 145-156.

16 Rizzo JA, Burgess P, Cartie RJ, Prasad BM. Moderate systemic hypothermia decreases burn depth progression. Burns 2013; 39: 436-444.

17 Kwon BK, Streijger F, Hill CE, Anderson AJ, Bacon M, Beattie MS et al. Large animal and primate models of spinal cord injury for the testing of novel therapies. Exp Neurol 2015; 269: 154-168.

18 Kwon BK, Stammers AM, Belanger LM, Bernardo A, Chan D, Bishop CM et al. Cerebrospinal fluid inflammatory cytokines and biomarkers of injury severity in acute human spinal cord injury. J Neurotrauma 2010; 27: 669-682.

19 Biglari B, Buchler A, Swing T, Biehl E, Roth HJ, Bruckner T et al. Increase in soluble CD95L during subacute phases after human spinal cord injury: a potential therapeutic target. Spinal Cord 2013; 51: 183-187.

20 Biglari B, Buchler A, Swing T, Child C, Biehl E, Reitzel T et al. Serum sCD95L concentration in patients with spinal cord injury. J Int Med Res 2015; 43: 250-256.

21 Ferbert T, Child C, Graeser V, Swing T, Akbar M, Biglari B et al. Tracking spinal cord injury: differences in cytokine expression of IGF-1, TGF- beta1 and SCD95L can be measured in blood samples and corresponds to neurological remission in a 12 week follow-up. J Neurotrauma 2016; 34: 607-614.

22 Moghaddam A, Heller R, Daniel V, Swing T, Akbar M, Gerner HJ et al. Exploratory study to suggest the possibility of MMP-8 and MMP-9 serum levels as early markers for remission after traumatic spinal cord injury. Spinal Cord 2016; 54: 957-960.

23 Moghaddam A, Sperl A, Heller R, Gerner HJ, Biglari B. sCD95L in serum after spinal cord injury. Spinal Cord 2016; 54: 957-960.

24 Moghaddam A, Sperl A, Heller R, Kunzmann K, Graeser V, Akbar M et al. Elevated serum insulin-like growth factor 1 levels in patients with neurological remission after traumatic spinal cord injury. PLoS ONE 2016; 11: e0159764.

25 Kirshblum SC, Burns SP, Biering-Sorensen F, Donovan W, Graves DE, Jha A et al. International standards for neurological classification of spinal cord injury (revised 2011). J Spinal Cord Med 2011; 34: 535-546.

26 Audigé L. Development and validation of a new generation for spine injury classification. Spine Classification and Severity Measures, 1st edn. Thieme: Stuttgart, 2009, pp 503-507.

27 Akaike H. Akaike's information criterion. International Encyclopedia of Statistical Science. Springer, 2011, pp 25.

28 R Development Core Team. R: A Language and Environment for Statistical Computing. R Foundation for Statistical Computing. Vienna, Austria, 2015

29 Robin X, Turck N, Hainard A, Tiberti N, Lisacek F, Sanchez JC et al. pROC: an opensource package for $\mathrm{R}$ and $\mathrm{S}+$ to analyze and compare ROC curves. BMC Bioinformatics 2011; 12: 77.

30 Wickham H. ggplot2: Elegant Graphics for Data Analysis. Springer-Verlag: New York, USA, 2009.

31 Moghaddam A, Muller U, Roth HJ, Wentzensen A, Grutzner PA, Zimmermann G. TRACP $5 \mathrm{~b}$ and CTX as osteological markers of delayed fracture healing. Injury 2011; 42: 758-764.

32 Müller ME, Nazarian S, Koch P, Schatzker J. The Comprehensive Classification of Fractures of Long Bones. Springer Science \& Business Media, 2012.

33 Zheng Y, Morris A, Sunkara M, Layne J, Toborek M, Hennig B. Epigallocatechin-gallate stimulates NF-E2-related factor and heme oxygenase-1 via caveolin-1 displacement. J Nutr Biochem 2012; 23: 163-168. 
34 Paul D, Ge S, Lemire Y, Jellison ER, Serwanski DR, Ruddle NH et al. Cell-selective knockout and $3 \mathrm{D}$ confocal image analysis reveals separate roles for astrocyte-and endothelial-derived CCL2 in neuroinflammation. J Neuroinflamm 2014; 11: 10.

35 Roberts TK, Eugenin EA, Lopez L, Romero IA, Weksler BB, Couraud PO et al. CCL2 disrupts the adherens junction: implications for neuroinflammation. Lab Investig 2012; 92: 1213-1233.

36 Mojsilovic-Petrovic J, Callaghan D, Cui H, Dean C, Stanimirovic DB, Zhang W. Hypoxia-inducible factor-1 (HIF-1) is involved in the regulation of hypoxia-stimulated expression of monocyte chemoattractant protein-1 (MCP-1/CCL2) and MCP-5 (Ccl12) in astrocytes. J Neuroinflamm 2007; 4: 12.

37 Mildner A, Mack M, Schmidt H, Bruck W, Djukic M, Zabel MD et al. CCR2+Ly-6Chi monocytes are crucial for the effector phase of autoimmunity in the central nervous system. Brain 2009; 132: 2487-2500.
38 Shechter R, Miller O, Yovel G, Rosenzweig N, London A, Ruckh J et al. Recruitment of beneficial M2 macrophages to injured spinal cord is orchestrated by remote brain choroid plexus. Immunity 2013; 38: 555-569.

39 Bank M, Stein A, Sison C, Glazer A, Jassal N, McCarthy D et al. Elevated circulating levels of the pro-inflammatory cytokine macrophage migration inhibitory factor in individuals with acute spinal cord injury. Arch Phys Med Rehabil 2015; 96 : 633-644.

40 Kiguchi N, Maeda T, Kobayashi Y, Fukazawa Y, Kishioka S. Macrophage inflammatory protein-1alpha mediates the development of neuropathic pain following peripheral nerve injury through interleukin-1beta up-regulation. Pain 2010; 149: 305-315.

41 Quandt J, Dorovini-Zis K. The beta chemokines CCL4 and CCL5 enhance adhesion of specific CD4+ T cell subsets to human brain endothelial cells. J Neuropathol Exp Neurol 2004; 63: 350-362. 